\title{
Traumatismo cranioencefálico em um hospital de referência no estado do Pará, Brasil: prevalência das vítimas quanto a gênero, faixa etária, mecanismos de trauma, e óbito
}

\author{
Maria Luana Carvalho Viégas ${ }^{1}$, Edmundo Luís Rodrigues Pereira², \\ Amanda Amaral Targino', Viviane Gonçalves Furtado', Daniella Brito Rodrigues ${ }^{3}$ \\ Hospital Metropolitano de Urgência e Emergência (HMUE), Ananindeua, PA, Brasil.
}

\section{RESUMO}

Objetivo: O objetivo deste estudo foi analisar pacientes vítimas de traumatismo cranioencefálico (TCE) atendidos em hospital de referência em traumatologia do Pará, Brasil, com descrição da prevalência de aspectos clínico-epidemiológicos como gênero, idade, faixa etária, mecanismo de trauma e óbito. Método: Estudo epidemiológico, transversal, observacional, descritivo e individual, baseado na análise de 250 prontuários de vítimas de TCE internadas no Hospital Metropolitano de Urgência e Emergência (HMUE), Ananindeua, PA, no período de janeiro de 2007 a março de 2008. Resultados: Predominou o gênero masculino (88\%), na faixa etária dos 20-30 anos de idade (32,4\%); o principal mecanismo de trauma foram os acidentes de tráfego (36,4\%), com os motociclísticos representando $44 \%$ deles; o óbito ocorreu em 22\% dos casos. Conclusão: A maior parte das vítimas foi de adultos jovens, do gênero masculino, mais suscetível aos acidentes e à violência; as lesões ocorreram predominantemente por acidentes de tráfego, apontando para a maior necessidade de fiscalização e conscientização da população sobre a importância das medidas preventivas para se evitar a mortalidade por TCE, que neste estudo foi de $22 \%$.

\section{PALAVRAS-CHAVE}

Traumatismos craniocerebrais, acidentes de trânsito, epidemiologia.

\section{ABSTRACT}

Traumatic brain injury in a reference hospital in Para, Brazil: prevalence of victims about gender, age group, mechanisms of trauma, and death

Objective: Analyze traumatic brain injury patients treated in a reference hospital in traumatology in Para, Brazil, describing the prevalence of clinical and epidemiological aspects as gender, age, mechanism of injury and death. Method: This study was cross-sectional and observational, based on analysis of 250 medical records of victims of head injury admitted to Urgency and Emergency Metropolitan Hospital (HMUE), Ananindeua, PA, from January 2007 to March 2008. Results: Males predominated (88\%), aged $20-30$ years $(32.4 \%)$, the main mechanism of injury were traffic accidents $(36.4 \%)$, with the motorcycle representing $44 \%$ of them, death occurred in $22 \%$ of cases. Conclusion: Most victims were young adults, males more susceptible to accidents and violence, injuries occurred predominantly by traffic accidents, pointing to the need for greater surveillance and public awareness of the importance of preventive measures to avoid mortality from TCE, which in this study was $22 \%$.

\section{KEYWORDS}

Craniocerebral trauma, accidents traffic, epidemiology.

1 Discente da Faculdade de Medicina da Universidade Federal do Pará (UFPA), Belém, PA, Brasil.

2 Mestre em Neurociências, neurocirurgião no Hospital Metropolitano de Urgência e Emergência, docente da UFPA, Belém, PA, Brasil.

3 Discente de Medicina da Universidade do Estado do Pará (UEPA), Belém, PA, Brasil. 


\section{Introdução}

O traumatismo cranioencefálico (TCE) é qualquer agressão capaz de lesão anatômica ou comprometimento funcional do couro cabeludo, crânio, meninges ou encéfalo. ${ }^{1}$ Ocorre por lesão direta ao parênquima encefálico (arma branca ou de fogo) ou forças de impacto e inércia sobre o crânio e o encéfalo, gerando deformação, aceleração ou desaceleração, consequentemente comprimindo e destruindo estruturas vasculares e neuronais. ${ }^{2}$

Nos Estados Unidos, os traumas mecânicos são a quarta causa de morte, considerada a principal entre a faixa etária de 1 a 45 anos de idade, sendo $40 \%$ desses óbitos ocorridos por TCE. ${ }^{3}$ Neste país, em 2009, foi estimado que chegue a 500 mil por ano o número de novos casos de TCE. Desses, 50 mil vão a óbito antes de chegar ao hospital, de 15 a 20 mil morrem após o atendimento hospitalar e, dos 430 mil restantes, 50 mil apresentaram sequelas neurológicas de maior ou menor severidade. ${ }^{2}$

No Brasil, nos últimos 10 anos, constatou-se que traumas mecânicos deixaram inválidos mais de 1 milhão de pessoas, com destaque para os acidentes de trânsito. ${ }^{3}$ Além disso, segundo o Manual Merck, o traumatismo cranioencefálico, em comparação com qualquer outra lesão neurológica, é a principal causa de morte e invalidez entre os indivíduos com menos de 50 anos de idade. ${ }^{4}$ Apesar do predomínio de adultos jovens como vítimas de TCE, há também outros dois picos de incidência relevantes quanto à faixa etária, localizados nos dois extremos: as vítimas na primeira década de vida $\mathrm{e}$ os com mais de 60 anos de idade. ${ }^{3}$

Os principais mecanismos de trauma são os acidentes automobilísticos, os atropelamentos, os acidentes ciclísticos (ou outros veículos não motorizados) e motociclísticos, as agressões físicas, as quedas, as lesões por arma de fogo, entre outros. ${ }^{5,6}$

\section{Objetivo}

Analisar pacientes vítimas de traumatismo cranioencefálico atendidos em um hospital de referência em traumatologia do Pará, Brasil, com descrição da prevalência de aspectos clínico-epidemiológicos como gênero, idade, faixa etária, mecanismo de trauma e óbito.

\section{Método}

Estudo epidemiológico, transversal, observacional, descritivo e individual, baseado na análise de prontuários de uma população inicial de 394 pacientes que se enquadram como vítimas de traumatismo cranioencefálico internados no Hospital Metropolitano de Urgência e Emergência (HMUE), Ananindeua, PA, no período de janeiro de 2007 a março de 2008. Desses, foram selecionados aleatoriamente 250. A coleta de dados foi realizada nos meses de janeiro a março de 2010, no arquivo do HMUE, mediante autorização do departamento de pesquisa do hospital. Os dados foram obtidos por meio de um protocolo-padrão de pesquisa elaborado pelos autores deste estudo. Para inferências estatísticas e confecção de tabelas, utilizou-se o programa Microsoft Excel (versão 2007).

\section{Resultados}

A análise quanto ao perfil epidemiológico dos pacientes permitiu a constatação de que a maior parte das vítimas era de homens, $88 \%$ (220/250), jovens entre 20-30 anos (32,4\%). O principal mecanismo de trauma se relacionou aos acidentes de trânsito, incluindo os motociclísticos, por atropelamento e os automobilísticos $(36,4 \%, 91 / 250)$ (Tabela 1).

\begin{tabular}{lcc}
$\begin{array}{l}\text { Tabela } 1 \text { - Perfil pessoal dos pacientes vítimas de TCE atendidos } \\
\text { no HMUE-PA, no período de janeiro de } 2007 \\
\text { a março de } 2008\end{array}$ \\
\hline Perfil pessoal & $\mathbf{n}$ & $\%$ \\
\hline Gênero & & \\
Feminino & 30 & 12 \\
Masculino & 220 & 88 \\
Idade (anos) & & \\
$<20$ & 60 & 24 \\
$20-30$ & 81 & 32,4 \\
$30-40$ & 41 & 16,4 \\
$40-50$ & 25 & 10 \\
$50-60$ & 21 & 8,4 \\
$>60$ & 20 & 8 \\
Não informado & 2 & 0,8 \\
\hline
\end{tabular}

Os traumas cranianos estudados foram causados principalmente pelos acidentes motociclísticos, sendo a taxa de mortalidade geral da amostra igual a $22 \%$ (55/250); 12\% (30/250) das vítimas evoluíram para morte encefálica apesar da intervenção cirúrgica; $4 \%$ $(10 / 250)$ morreram durante o atendimento inicial no hospital; e os demais $(6 \%, 15 / 250)$ decorreram de complicações sistêmicas e/ou infecciosas nosocomiais (Tabela 2 e Figura 1). 


\begin{tabular}{|c|c|c|}
\hline \multicolumn{3}{|c|}{$\begin{array}{c}\text { Tabela } 2 \text { - Prevalência dos mecanismos de trauma dos } \\
\text { pacientes vítimas de TCE atendidos no HMUE-PA, } \\
\text { no período de janeiro de } 2007 \text { a março de } 2008\end{array}$} \\
\hline Mecanismo de trauma & $\mathbf{n}$ & $\%$ \\
\hline Motociclismo & 40 & 16 \\
\hline $\mathrm{PAF}^{*}$ & 36 & 14,4 \\
\hline Atropelamento & 35 & 14 \\
\hline Agressão física & 28 & 11,2 \\
\hline $\mathrm{FAB}^{\star *}$ & 27 & 10,8 \\
\hline Altura & 26 & 10,4 \\
\hline Trauma direto & 22 & 8,8 \\
\hline Automobilismo & 16 & 6,4 \\
\hline Outros & 20 & 8 \\
\hline Total & 250 & 100 \\
\hline
\end{tabular}

* Ferimento por arma de fogo (PAF); ${ }^{* *}$ Ferimento por arma

branca (FAB). Fonte: Banco de dados da pesquisa.

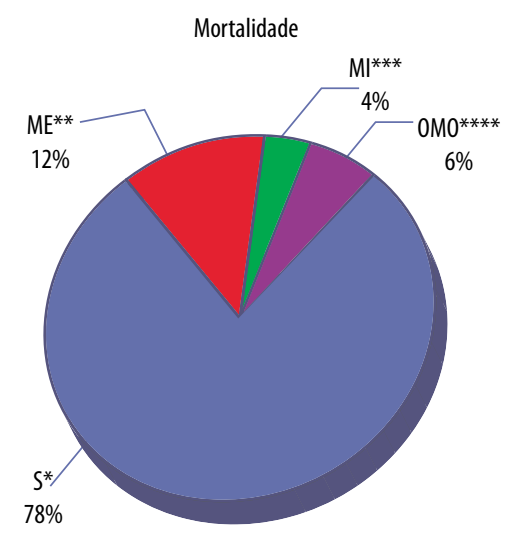

Figura 1 - Análise da evolução para a mortalidade dos pacientes vítimas de TCE atendidos no HMUE-PA, no período de janeiro de 2007 a março de 2008.

*Sobrevida (S); ${ }^{* *}$ Morte encefálica (ME); ${ }^{* * *}$ Morte imediata

(MI); ${ }^{* * * *}$ Outros mecanismos de óbito (OMO). Fonte: Banco de dados da pesquisa.

\section{Discussão}

Em estudo realizado em 2004, a partir da análise de 555 prontuários de pacientes vítimas de traumatismo cranioencefálico em hospital de referência da Bahia, detectou-se predomínio do gênero masculino em $83 \%$ dos casos, estando a maior parte dos pacientes na faixa etária compreendida entre 21 e 30 anos $(23,2 \%) .^{3}$ No hospital da presente pesquisa, $88 \%(\mathrm{n}=30)$ das vítimas eram de indivíduos do gênero masculino, contra um percentual inferior de mulheres $(12 \% / \mathrm{n}=230)$. Predominaram indivíduos entre os 20 e 30 anos de idade, com prevalência de $32,4 \%(n=81)$, seguida por jovens com idade inferior a 20 anos, indicando o TCE ser um dos principais fatores de morbimortalidade por causas externas na faixa etária mais nova da população. Tal distribuição também foi analisada em outro estudo ${ }^{7}$ realizado em São Paulo, em 2008, em que houve predomínio da faixa etária entre os 16 e os 30 anos, o adulto jovem, entre as vítimas de TCE, sendo a inexperiência e a imprudência prováveis justificativas de tal envolvimento.

Nos Estados Unidos ${ }^{8}$ trabalhos que analisam os aspectos epidemiológicos e as complicações cirúrgicas do traumatismo cranioencefálico apontam média de idade igual a 45 anos, embora os homens continuem sendo os mais acometidos ( $80 \%$ dos casos). Essa diferença de faixa etária predominante com relação ao presente estudo (maioria de 20-30 anos) pode decorrer das diferenças entre as leis de tráfego e a organização/ fiscalização do Brasil, em relação aos Estados Unidos, uma vez que lá os jovens começam a dirigir mais tardiamente, aliado a maior punição e incorruptibilidade das forças de fiscalização.

Cardoso et al. ${ }^{1}$ afirmam que, dentre todas as causas, o principal responsável por traumas cranianos é o acidente de tráfego, sendo apontado como preponderante $(36,4 \% / \mathrm{n}=91)$ no mecanismo de trauma do presente estudo. Estão entre os mais comuns acidentes os motociclísticos $(16 \% / \mathrm{n}=40)$, por atropelamento $(14 \% / \mathrm{n}=$ $35)$ e automobilísticos $(6,4 \% / \mathrm{n}=16)$. Esse fato se deve à alta velocidade, à falta de atenção, ao alcoolismo, ao não uso de equipamentos de proteção e à falta de fiscalização e mau planejamento das vias de tráfego, fatores verificados por estudo em grandes cidades brasileiras, como São Paulo e Brasília, onde houve destaque primeiramente para a colisão entre veículos, seguida dos atropelamentos. $^{3}$

Esse mecanismo de agressão deve ser analisado com atenção, pois acidentes automobilísticos são as causas mais graves de lesões no crânio e na face, gerando elevada incapacidade/mortalidade dos pacientes. Provavelmente, isso ocorre em virtude de os equipamentos de segurança para a proteção individual, como o cinto e o airbag, não serem totalmente seguros, ou ainda por não serem utilizados pela maioria da população. ${ }^{7}$

Violência urbana e agressões físicas são causas crescentes de trauma mecânico em grandes metrópoles. ${ }^{3}$ Foi verificado na presente pesquisa que $14,4 \%(n=36)$ das internações por TCE ocorreram por ferimentos com armas de fogo e, ainda, $11,2 \%(\mathrm{n}=28)$ foram ocasionados por agressão física direta, evidenciando a importância da violência urbana como causa de traumatismo cranioencefálico.

$\mathrm{Na}$ região amazônica, deve-se dar atenção para os casos de quedas de alturas ocorridos em 10,4\% ( $\mathrm{n}=$ 26), em sua maioria relacionados à atividade de extrativismo vegetal desenvolvida no interior do estado do Pará. Outros estudos analisados ${ }^{3}$ demonstram que a 
frequência dessas quedas depende essencialmente do meio e da população estudados, vinculados também ao nível socioeconômico e ocupacional da população.

Uma dessas pesquisas ${ }^{9}$ investigou a evolução de pacientes vítimas de TCE grave atendidos em hospital de referência de Campinas no ano de 2004, tendo encontrado 75 falecimentos $(36,40 \%), 38(18,45 \%)$ receberam alta sem responder a comandos verbais simples e $48(23,30 \%)$ saíram do hospital respondendo normalmente aos comandos verbais. As vítimas de TCE atendidas no hospital do presente estudo apresentaram taxa de mortalidade igual a $22 \%$, sendo $12 \%$ por morte encefálica e $10 \%$ por outros mecanismos de falecimento. As diferenças entre as taxas de morte observadas podem ser atribuídas ao fato de a pesquisa de Campinas ter selecionado previamente pacientes com TCE grave e, portanto, com mau prognóstico, enquanto o presente estudo não fez essa seleção. Em inquérito epidemiológico sobre a morbimortalidade por TCE na cidade de São Paulo em 2000, ${ }^{10}$ ocorreram $10,2 \%$ de óbitos entre a população analisada, a qual não estava estratificada pela gravidade das lesões.

\section{Conclusão}

A maior parte das vítimas de TCE se constitui de adultos jovens e do gênero masculino, mais suscetível à violência, à imprudência e aos acidentes de trabalho. Dentre as causas do trauma, destacam-se os acidentes com veículos por causa da alta velocidade e da falta de atenção.

Os dados revelam a necessidade de programas voltados à faixa etária entre os 20-30 anos que aborde a necessidade da prevenção pessoal diante do perigo de morbimortalidade das lesões traumáticas.

Sendo assim, conclui-se que é de grande importância a elaboração de políticas de prevenção mais direcionadas e um atendimento mais eficaz ao paciente traumatizado, priorizando-se as medidas de conscientização e fiscalização do trânsito, a fim de reduzir os acidentes por tráfego.

\section{Referências}

1. Cardoso OB, Franco MM, Gusmão SNS. Traumatismo cranioencefálico no adulto. In: Pires MTB, Starling SV. Manual de urgências em pronto-socorro. $8^{\mathrm{a}}$ ed. Rio de Janeiro: Guanabara Koogan; 2006. p. 323-5.

2. Andrade AF, Marino RJR, Brock RS, Rodrigues JC, Masini M. Diagnóstico e conduta no paciente com traumatismo cranioencefálico moderado e grave por ferimento por projétil de arma de fogo. São Paulo: Associação Médica Brasileira e Conselho Regional de Medicina; 2004. p. 15.

3. Melo JR, Silva RA, Moreira ED Jr. Characteristics of patients with head injury at Salvador City (Bahia, Brazil). Arq Neuropsiquiatr. 2004;62(3A):711-4.

4. Berkow R, Beers MH. Manual Merck de medicina: diagnóstico e tratamento. $17^{\mathrm{a}}$ ed. São Paulo: Roca; 2001.

5. Bruns J Jr, Hauser WA. The epidemiology of traumatic brain injury: a review. Epilepsia. 2003;44(Suppl 10):2-10.

6. Maldaun MV, Zambelli HJ, Dantas VP, Fabiani RM, Martins $\mathrm{AM}$, Brandão $\mathrm{MB}$, et al. Analysis of 52 patients with head trauma assisted at pediatric. Intensive Care Unit: considerations about intracranial pressure monitoring. Arq Neuropsiquiatr. 2002;60(4):967-70.

7. Nascimento EN, Gimeniz-Paschoal SR. The human accidents and their implications for functional communication: opinions of teachers and students about higher education. Ciênc Saúde Coletiva. 2008;13(Suppl 2):2289-98.

8. Tallon JM, Ackroyd-Stolarz S, Karim SA, Clarke DB. The epidemiology of surgically treated acute subdural and epidural hematomas in patients with head injuries: a population-based study. Can J Surg. 2008;51(5):339-45.

9. Dantas Filho VP, Falcão AL, Sardinha LA, Facure JJ, Araújo $S$, Terzi RG. Relevant factors influencing the evolution of 206 patients with severe head injury. Arq Neuropsiquiatr. 2004;62(2A):313-8.

10. De Sousa RM, Regis FC, Koizumi MS. Traumatic brain injury: differences among pedestrians and motor vehicle occupants. Rev Saude Publica. 1999;33(1):85-94.

Endereço para correspondência

Maria Luana Carvalho Viégas

Av. José Bonifácio 2464, casa 5, Guamá

66065-362 - Belém, PA, Brasil

E-mail: mluanaviegas@gmail.com 